\title{
NIOSOMES AS AN APPROACH TO IMPROVE THE SOLUBILITY AND BIOAVAILABILITY OF BCS CLASS II DRUGS
}

\author{
GAURANG SAWANT ${ }^{1}{ }^{*}$, GEETA BHAGWAT ${ }^{1}$
}

${ }^{1}$ Department of Pharmaceutics, H. K. College of Pharmacy, Oshiwara, Mumbai, India

*Email: sawant.gaurang10@gmail.com

Received: 01 Dec 2020, Revised and Accepted: 30 Jan 2021

\begin{abstract}
Based on their solubility and permeability, drugs are typically divided into four classes (Classes I-IV) according to the biopharmaceutics classification system (BCS). Of these classes, BCS class II drugs have high permeability and low solubility; not only do these characteristics constitute the rate-limiting step in the formulation of these drugs but the low solubility in water results in low bioavailability. Thus, methods for improving their solubility have been developed using lipid carriers such as liposomes, niosomes, and aquasomes; other approaches include self-microemulsifying drug delivery systems (SMEDDS) and self-nano-emulsifying drug delivery systems (SNEDDS). Currently, niosome-based drug delivery systems that utilize nonionic surfactants, drugs, and cholesterol in varying ratios are being widely used to deliver both hydrophilic and lipophilic drugs in addition to several other applications of niosomes.
\end{abstract}

Keywords: Biopharmaceutics, Solubility, Permeability, Lipid carriers, Liposomes, Niosomes

(C) 2021 The Authors. Published by Innovare Academic Sciences Pvt Ltd. This is an open access article under the CC BY license (https://creativecommons.org/licenses/by/4.0/) DOI: https://dx.doi.org/10.22159/ijap.2021v13i2.40423. Journal homepage: https://innovareacademics.in/journals/index.php/ijap

\section{INTRODUCTION}

The biopharmaceutics classification system (BCS) is a useful mechanism used by researchers for obtaining biowaivers during in vivo bioequivalence studies and for decision making when determining the required solubility and permeability during drug discovery. This is because BCS is established upon a scientific blueprint highlighting the three rate-limiting steps critical in oral absorption: the liberation of the drug from its dosage form [1], prolongation of the dissolved state along with the whole gastrointestinal (GI) tract [2], and penetration of the drug molecules via the GI membrane into the blood [3]. Additionally, enterohepatic metabolism constitutes a fourth step that affects the systemic accessibility along with the release of metabolites into systemic blood circulation. The biopharmaceutical drug disposition classification system (BDDCS) proposed by Wu and Benet comprehensively describes the absorption operation by including the fourth rate-limiting step of first-pass metabolism. Niosomes are uncharged surfactant vesicles comprising microscopic lamellar structures built upon an amalgamation of uncharged surfactants such as the alkyl or dialkyl polyglycerol ether class and cholesterol formed via subsequent hydration in an aqueous buffer [2]. In niosomes, the vesicle-building amphiphiles are uncharged surfactants (e. g., Span 60) that are typically balanced by incorporating cholesterol and minuscule amounts $[1.5 \mathrm{mg}]$ of a negatively charged surfactant such as dicetyl phosphate [4]. Several methods exist for niosome fabrication such as ether injection $[5,6]$, the hand-shaking method [6], sonication [6], microfluidization [7], reverse phase evaporation [8], the bubble method [9], multiple membrane extrusion [7], and the proniosomal approach [10]. Characterization parameters include particle size, in vitro drug release, entrapment efficiency, and drug content together with some specific characteristics that depend on the formulation mechanism such as skin permeation etc. Herein, a literature survey was performed using accessible databases such as Google Scholar, PubMed, and Scopus to review research articles and thus compile a comprehensive yet concise introduction to the BCS and niosomes along with their applications.

\section{Biopharmaceutics classification system}

BCS is a scientific framework for classifying a drug based on its aqueous solubility and intestinal penetrability [11]. When used in conjunction with the in vitro dissolution properties of the concerned drug, BCS considers three important parameters: solubility, intestinal permeability, and dissolution rate. Together these parameters determine the essential factors of the speed and limit of oral drug absorption from immediaterelease (IR) solid oral dosage forms [12, 13]. Based on the BCS framework, the drugs can be classified into four basic groups using the criteria of their solubility and permeability toward gastrointestinal tract (GIT) mucosa, as shown in fig. 1. The solubility categorization of a drug in the BCS is determined on the basis of the maximum dosage strength of the IR product. A drug is deemed highly soluble when its maximum dosage strength is soluble in a minimum of $250 \mathrm{ml}$ of water-based media spanning a $\mathrm{pH}$ range of 1.0-7.5; otherwise, the drug is deemed a poorly soluble candidate. The volume approximation of $250 \mathrm{ml}$ was established in the literature using traditional bioequivalence study methods $[12,13]$. The permeability classification is directly based on a drug's intestinal absorption limit in humans or indirectly based on the calculations of mass transfer speed via the human intestinal membrane. A drug is deemed highly permeable when the intestinal absorption limit is $\geq 90 \%$. Otherwise, the drug is deemed poorly permeable $[12,13]$. An IR drug is categorized as a fast dissolution product when at least $85 \%$ of the stated amount of the drug dissolves in less than $30 \mathrm{~min}$ when utilizing the United States Pharmacopoeia (USP) Apparatus I set at 100 rotations per minute (rpm) or USP Apparatus II at $50 \mathrm{rpm}$ comprising a minimum volume of $900 \mathrm{ml}$ of each of the following media: 1) acidic media, such as $0.1 \mathrm{~N}$ hydrochloric acid or USP simulated gastric fluid with an absence of enzymes; 2) a pH 4.5 buffer, and 3) a pH 6.8 buffer or USP simulated intestinal fluid in the absence of enzymes. Otherwise, the drug is deemed a slow dissolution product.

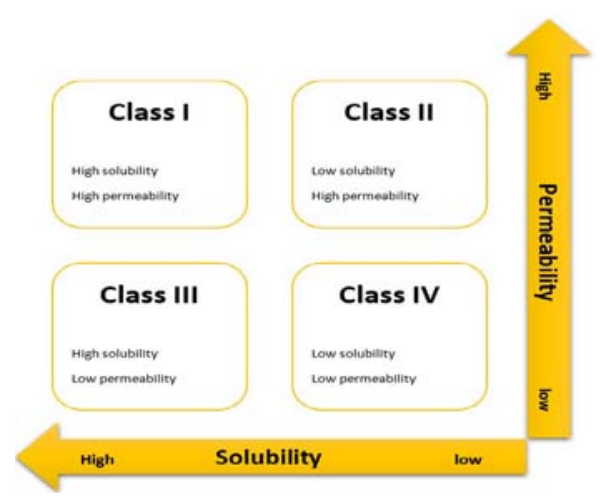

Fig. 1: Biopharmaceutics classification system ${ }^{\dagger \dagger}$ Adapted from commons. wikimedia.org, by MKD 2020

https://commons.wikimedia.org/wiki/File:Biopharmaceutics Classification_System_(BCS).jpg. Copyright by, MKD 2020,

\#Copyright permission obtained under license CC BY 4.0 (Creative Commons Attribution-Share Alike 4.0 International) 
Solubility and permeability measurement in the discovery/ development settings

Drug discovery begins with the recognition of a pharmacophore by scanning a library of recombinant chemical series through a biological method such as attachment to a receptor or enzyme blocking. The prototypical hit compounds are then directed toward high throughput screening (HTS) pharmaceutical profiling. Compounds with the required biological and pharmaceutical resources are subsequently examined in vivo for confirming the mechanism of action followed by lead optimization via chemical synthesis [14].

\section{Solubility determination}

HTS solubility examination typically begins with a dimethyl sulfoxide (DMSO) solution. This stock solution is incorporated into a pH 7 phosphate buffer with a volume of one microliter at all times until the compound precipitates out of the solution [15]. The light scattering phenomenon observed from the precipitated material is detected using an ultraviolet (UV) detector or directly via laser nephelometry [16]. Another method implements equilibration, followed by filtration of the DMSO-buffer suspension. Then, the filtrate is analyzed through a direct UV method. These automatic solubility procedures are currently being commercially used for HTS [17]. Precipitation of the compound from the DMSO stock solution is based on the rate and extent of nucleation occurring within the solution and hence demonstrates kinetic solubility. However, equilibrium solubility is mandatory by the Food and Drug Authority FDA for BCS classification. The shake flask method is a nonautomatic method used to compute equilibrium solubility at various $\mathrm{pH}$ levels. The $\mathrm{pH}$ solubility profiling is usually performed only after the lead candidate has been selected. The kinetic solubilities are normally parallel but slightly higher than the equilibrium solubility.

\section{Permeability determination}

HTS permeability analysis has been used worldwide with 24 automated wells of the tissue-cultured human colon adenocarcinoma (Caco-2) cell system [18]. This system comprises a monolayer of cells that are allowed to develop onto a filter acting as support, which separates the drug donor and acceptor compartments. Permeability is examined by analyzing drug arrival in the acceptor compartment using the direct UV or liquid chromatography-mass spectrometry method. Although the Caco-2 cell system is an automated and well-established HTS method, it suffers from certain limitations. For compounds delivered through the passive transcellular course, the Caco-2 permeability is an appropriate procedure for forecasting human permeability. For compounds delivered via paracellular or transporter-governed processes or for highly insoluble compounds, the Caco-2 permeability tends toward miscalculation of human permeability because of three reasons: (1) excessive expression of p-glycoprotein (Pgp) efflux pumps, (2) lessening of the paracellular transport route owing to the absence of liquid pores, and (3) random attachment of insoluble compounds onto the filter support and plastic components thereby decreasing visible penetrability [19]. As a compound advances from discovery to the development stage, more tedious pressing procedures, e. g., the in situ rat gut perfusion method, are employed to rectify the false-negative conclusions produced by the Caco-2 method [20]. The compounds utilized in these tedious procedures are again characterized by low (BCS classes III and IV) to high permeability (BCS classes I and II) when entering the development stage. Moreover, the Caco-2 cells require $21 \mathrm{~d}$ to fully develop into a monolayer of integrity, whereas other cells such as the Madin-Darby canine kidney (MDCK) cell line require only 3-7 d; hence, the latter have been used more frequently to accelerate the analysis [21]. However, MDCK cells exhibit the same problems as the Caco-2 cells in terms of generating false-negative results and thus could represent low permeability. In addition, the MDCK cells present more issues than Caco-2 cells including less expression of various efflux pumps and failure to meet the criteria for screening of chemical series with identified efflux problems. Both the in vitro Caco-2/MDCK cell lines method and the in situ rat perfusion methods are trusted by the FDA in terms of BCS classification.

\section{Niosomes}

Niosomes are microscopic layered structures of 10-1000-nm size, and their core is environmentally friendly and non-reactive toward the human immune system and biocompatible surfactants [22]. The niosomes are amphipathic, i.e., a water-soluble drug can be locked in their core cavity region and water-insoluble drugs in the non-polar region are present inside their bilayer; hence, both water-soluble and water-insoluble drugs can be added into niosomes as shown in fig. 2. Structurally, niosomes are similar to liposomes: they possess the same drug delivery potential and offer more chemical stability than liposomes at lower production costs. Both vesicles comprise a bilayer, which is composed of uncharged surfactants in the case of niosomes and of phospholipids in the case of liposomes.

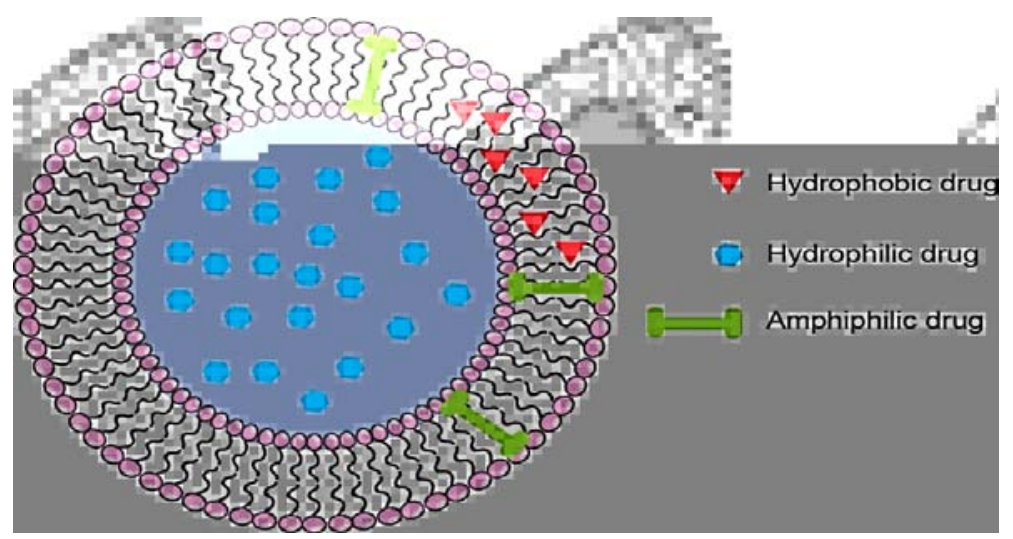

Fig. 2: Structure of niosome ${ }^{+\neq,+}$Adapted from Reference [23], *Copyright License obtained under CC BY license (https://creativecommons.org/licenses/by/4.0/)

\section{Preparation methods}

\section{Ether injection method}

The primitive step in niosome formulation via the ether injection method involves surfactant dissolution in any volatile solvent such as diethyl ether, chloroform, or methanol. The solution is then incorporated into an aqueous drug solution via injection using a 14 Gauze needle maintained at $60^{\circ} \mathrm{C}$ on a water bath or on a magnetic stirrer. Consequently, monolayered vesicles with sizes ranging from 50 to $1000 \mathrm{~nm}$ are produced through the volatile solvent's atomization [24].

\section{Hand-shaking method}

The hand-shaking method, also known as the thin-film hydration technique, involves the dissolution of the surfactant and cholesterol in a volatile organic solvent and subsequent transfer into a rotary 
evaporator. Following evaporation, a thin layer of solid remains on the wall of the flask. This dried layer is then rehydrated using an aqueous phase of the drug of interest. Alternatively, this procedure can be performed at room temperature via light agitation [6, 25].

\section{Sonication}

Niosomes can be fabricated by sonicating an amalgamation of surfactant, cholesterol, and an aqueous phase containing the drug maintained at $60{ }^{\circ} \mathrm{C}$ for $3 \mathrm{~min}$ in a beaker placed in a probe or bath sonicator. The vesicles thus formed have less particle size exhibit size uniformity $[6,25]$.

\section{Microfluidization}

Microfluidization is another duplicable method that yields size uniformity via operating, i.e., two fluidized streams flowing forward and intersect with each other at ultrahigh speeds through an accurately defined microchannel $[24,25,7]$.

\section{Reverse-phase evaporation method}

The reverse-phase evaporation method utilizes an amalgamation of surfactant and cholesterol in a 1:1 ratio in addition to ether and chloroform. An aqueous phase containing the target drug is incorporated into the concoction followed by sonication at a temperature of $4{ }^{\circ} \mathrm{C}-5{ }^{\circ} \mathrm{C}$. Sonication is continued for about $5 \mathrm{~min}$ after incorporating about $10 \mathrm{ml}$ of phosphate-buffered saline into the concoction. The organic solvent is atomized at $40{ }^{\circ} \mathrm{C}$ under low pressure, and the persisting suspension is thinned using phosphatebuffered saline. The amalgamation is heated at $60{ }^{\circ} \mathrm{C}$ for $10 \mathrm{~min}$, and the ultimate product of niosomes is attained [24, 25, 8]. Fig. 3 shows a schematic of this method.

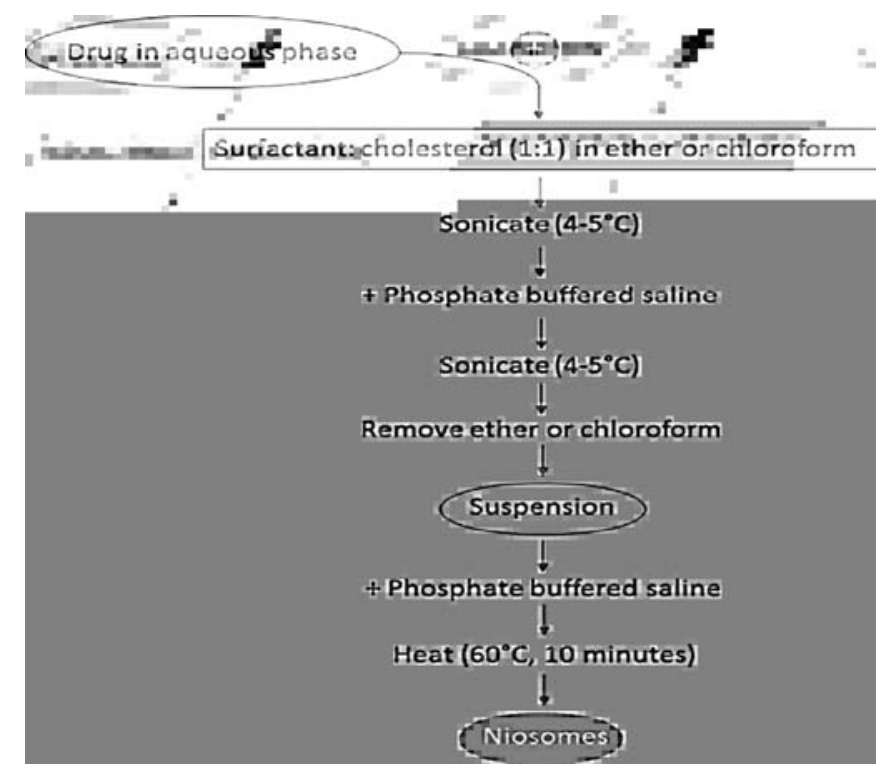

Fig. 3: Reverse-phase evaporation method for the preparation of niosomes ${ }^{\dagger \ddagger},{ }^{\dagger}$ Adapted from Reference [26], $\neq$ Copyright permission obtained under CC BY License (https://creativecommons.org/licenses/by-nc-nd/4.0/)

\section{The bubble method}

Niosomes can also be fabricated in the absence of organic solvents through the bubble method, wherein a bubbling unit containing a roundbottomed flask with three necks is placed in a water bath; a water-cooled reflux condenser and thermometer are placed in the first and second necks, respectively, whereas nitrogen gas is introduced through the third neck. Surfactant and cholesterol amalgamated at $70{ }^{\circ} \mathrm{C}$ in a buffer are blended and bubbled at $70{ }^{\circ} \mathrm{C}$ by introducing nitrogen gas into the apparatus $[24,9]$. Fig. 4 shows a schematic of this method.
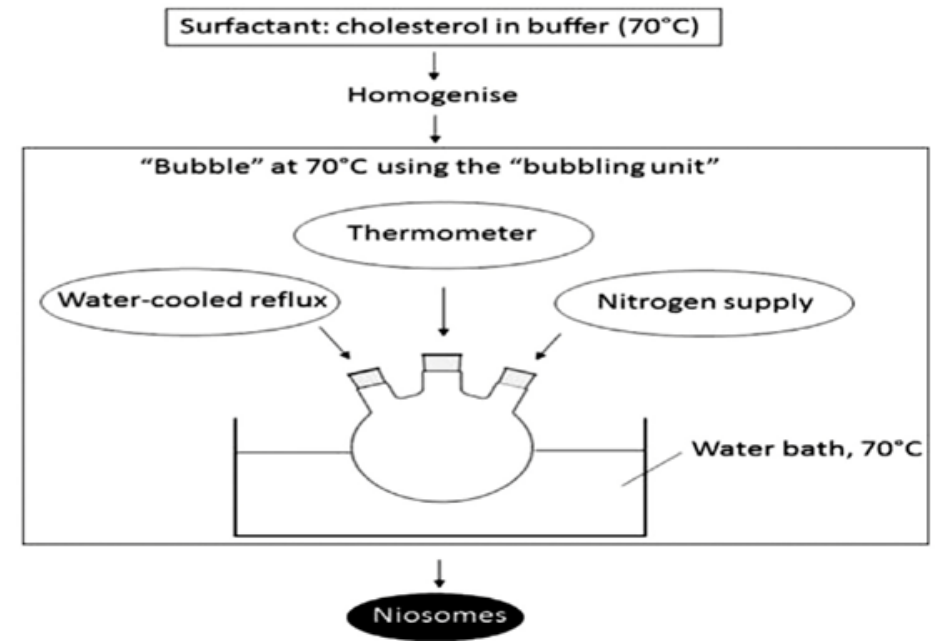

Fig. 4: The Bubble Method for Preparation of Niosomes ${ }^{\dagger \neq},{ }^{\dagger}$ Adapted from Reference [26], ${ }^{\ddagger}$ Copyright permission obtained under CC BY License (https://creativecommons.org/licenses/by-nc-nd/4.0/) 


\section{Multiple membrane extrusion method}

In the multiple membrane extrusion method an amalgamation of surfactant, cholesterol, and dicetyl phosphate is dissolved in chloroform, and the resulting concoction is vaporized to form a thin film. This film is dampened with an aqueous drug solution, and the resulting suspension is extruded using polycarbonate membranes, which are inserted in series to create a maximum of 8 passages [24, 25, 27].

\section{Proniosomal method}

In this niosome fabrication method, a water-soluble transporter such as sorbitol is sprayed with a surfactant to form a dry formulation in which each water-soluble particle is laminated with a thin layer of dry surfactant. This formulation is labeled as a proniosome. The proniosome powder thus formed is subsequently loaded into a screw-capped vial, and blended with water or saline at $80{ }^{\circ} \mathrm{C}$ by vortexing. This is followed by stirring for about $2 \mathrm{~min}$, thus producing the final niosomal suspension [28].

\section{Niosome evaluation}

\section{Entrapment efficiency}

For assessing a drug's entrapment efficiency within the niosomes, any of the following three methods can be used: centrifugation, gel filtration, or complete vesicle disruption using $50 \% \mathrm{n}$-propanol or $0.1 \%$ Triton $\mathrm{X}-100$. This method estimates the quantity of the drug remaining locked inside the niosomes and examines the final solution through an assay method suitable for the target drug. In this method, entrapment efficiency (EF) can be defined as follows [29].

$$
\text { Percentage drug entrapment }(\%)=\frac{[\text { Total Drug Content }(\mathrm{mg})-[\text { Free Drug Content }(\mathrm{mg})]}{100} \times
$$

\section{Drug content}

The fabricated niosomal formulations are transferred into a test tube in which $10 \mathrm{ml}$ of methanol is introduced to break down the niosomes followed by the destruction of the outer membrane, thereby liberating the entrapped drug. The quantity of the released drug can be estimated using a UV spectrophotometer at a specified wavelength, thus enabling the calculation of the total mass of drug present in the formulation [30,31].

\section{In vitro drug release}

The in vitro release rate can be determined using a dialysis tube. In this method, a dialysis sac is cleansed and immersed in distilled water. The niosome suspension is transferred using a pipette inside a bag composed of the dialysis tubing and fastened; then the bag is left in a $200 \mathrm{ml}$ buffer solution placed in a $250 \mathrm{ml}$ beaker and subjected to continuous vibration at $25^{\circ} \mathrm{C}$ or $37^{\circ} \mathrm{C}$. The drug content in the resulting buffer solution is assessed at various time points via a suitable assay method [32].

\section{Particle size and morphology}

The most commonly used methods for determining particle size and the morphology of niosomes are dynamic light scattering (DLS) [33], scanning electron microscopy (SEM) [34], transmission electron microscopy (TEM) [35], freeze-fracture replication-electron microscopy (FF-TEM) [36], and cryotransmission electron microscopy (cryo-TEM) [37]. DLS simultaneously provides detailed information on particle size and critical information on the solution's homogeneity. A single sharp peak in the DLS result indicates the presence of a single population of scatters. The polydispersity index (PI) is a useful parameter in this regard. If $\mathrm{PI}<0.3$, then homogeneous population exists in the colloidal system and if PI>0.3 then the system is not homogeneous [33]. The abovementioned microscopic methods are commonly used to evaluate niosome morphology.

\section{Niosome applications}

\section{Sustained release}

A constant concentration of drugs possessing a narrow therapeutic index and low water solubility can be maintained in the blood plasma via niosomal encapsulation, thus achieving sustained release action. Azmin et al. [38] Proposed using part of the liver as a storage site for the absorption of methotrexate after niosome administration by the liver cells, thereby realizing sustained drug release.

\section{Localized drug action}

Niosomal dosage is an attractive method to realize localized drug action at the administration site because of the size of niosomes and their lesser penetrability compared with liposomes epithelium and connective tissue. This improves the drug's effectiveness and reduces its systemic toxic effects. For example, antimonials entrapped within niosomes are absorbed by mononuclear cells resulting in localized drug action, potency enhancement, and dual reduction in dosage as well as toxicity $[9,39]$.

\section{Cancer}

Common limitations of cancer chemotherapy manifest as side effects and less therapeutic efficacy. Doxorubicin, a broad-spectrum anthracycline used for anticancer activity, has demonstrated a dosedependent irreversible cardiotoxic effect [4]. However, when this drug was administered in the form of niosomes to mice possessing an S-180 tumor, the lives of the mice were prolonged and the multiplication of sarcoma was reduced. This can be attributed to the high effectiveness of niosomes in drug entrapment, resulting in sustained blood circulation and changes in drug metabolism [4, 40]. Another popular anticancer drug, daunorubicin hydrochloride, demonstrated increased anticancer activity in its niosomal entrapped form when compared against the effectiveness of the drug alone. The niosomal formulation constructively shattered Dalton's ascitic lymphoma cells in a short duration. In the case of bleomycin, a potent anticancer drug, compared with its free drug form, the form of the drug entrapped within niosomes comprising $47.5 \%$ cholesterol accumulated in high levels at the tumor position [41]. Methotrexate is a well-proven toxic synthetic antineoplastic drug used in chemotherapy, either alone or in conjunction with other medications, to cure different types of cancers. Extensive research has demonstrated that intravenous administration of methotrexate entrapped in niosomes to S-180 tumor-possessing mice causes total retrogradation of the tumor, relaxed drug clearance, and an elevated plasma level of methotrexate [42]. Improved drug penetration was observed when 5-fluorouracil was developed in bola-surfactant niosomes to cure skin cancer [43]. In addition to the fact that boosted antitumor activity is observed, in some situations, drug entrapment in niosomal vesicles reduces the cytotoxicity toward normal cells, as reported in a study on the design of niosomes containing vincristine. Frequent side effects of the drug, such as neurological toxicity, diarrhea, and alopecia, were reduced, whereas antitumor activity was increased in a mouse model of S-180 sarcoma after niosome-entrapped vincristine was administered [44]. Tocotrienol was the foremost drug to be reported for its anticancer activity in the initial years of the 1990s, and was subsequently entrapped in niosomes by Fu et al. [45]. Applying the film hydration method doubled (at minimum) the cytotoxic effect of niosomal tocotrienol in shattering breast cancer cells with the cells' drug absorption improving 2.5 -fold. The antitumor activity of the preparation was also observed in female BALB/c nude mice carrying breast cancer cells [46]. Curcumin is well known to demonstrate many therapeutic uses, including anticancer properties [47]. A new niosome system comprising Span 80 , Tween 80 , and poloxamer 188 was demonstrated to have excellent encapsulating efficiency (92.3\%) with respect to curcumin. When niosomal curcumin was incorporated into ovarian cancer A2780 cells, it increased cytotoxic and apoptotic activities as compared with freely used curcumin. This can be attributed to the properly controlled liberation of curcumin from niosomes [48]. Sharma et al. [49] Fabricated niosomes using Tween 80 and cholesterol through a film hydration method. Two anticancer drugs, curcumin and doxorubicin hydrochloride, were entrapped within the formulated niosomes; while curcumin was found to be accumulated in the shell, doxorubicin hydrochloride was found to be accumulated in the inner aqueous core of the niosomes. Higher cytotoxicity toward cervical cancer cells (HeLa cells) was observed for the double-drug-encapsulated niosomes. Artemisinin, which is extracted from the Chinese herb Artemisia annua, is frequently used in the treatment of fevers and chills [50]. The herb 
also has antitumor properties [51]. However, the use of artemisinin is limited because of its low solubility in water and oil coupled with poor bioavailability. Moreover, it possesses a short half-life in humans [52]. To enhance the effectiveness of artemisinin, Dwivedi $e t$ al. [53] entrapped the compound within nanovesicular niosomes. They demonstrated that the entrapped artemisinin showed cytotoxicity toward melanoma cells with little to no toxicity toward normal skin cells, hinting at artemisinin's potential therapeutic use in melanoma treatment. Tamoxifen citrate is recognized as a hormone antagonist administered to breast cancer patients having estrogen receptor-positive tumors [54].

However, conditions such as localization, effectiveness, providing sustained drug release, and reducing side effects of drugs are significant issues encountered in this type of cancer treatment. Nevertheless, Shaker et al. [55] encapsulated the drug into niosomes and assessed its cellular uptake in addition to its cytotoxicity and in vivo effectiveness. Niosomal tamoxifen demonstrated increased cellular uptake coupled with higher cytotoxicity against the MCF-7 breast cancer cell line and showed increased regression in in vivo tumor volume. Mitoxantrone has been utilized in chemotherapy for various cancers such as leukemia, lymphoma, breast and prostate cancers, and multiple sclerosis. However, the administration of this drug is commonly followed by serious systemic toxicity, mainly cardiotoxicity. Tila et al. [56] prepared $\mathrm{pH}$-sensitive, polymermodified, and plasma-stable niosomes to deliver this drug. The cytotoxicity of mitoxantrone niosomes was examined against human ovarian cancer (OVCAR-3), human breast cancer (MCF-7), as well as human umbilical vein endothelial cell lines. Mitoxantrone entrapped in the $\mathrm{pH}$-sensitive niosomes demonstrated increased cytotoxicity when compared against traditional niosomes on the cancer cells, but showed lesser cytotoxic activity when used on the endothelial cell line. These results demonstrate niosomal formulations to be promising carriers in minimizing the side effects of mitoxantrone. Cisplatin, a commonly used anticancer drug, acts by starting apoptosis and necrosis of the cells.

However, the use of cisplatin is accompanied by various harmful side effects, mainly nephrotoxicity and neurotoxicity [57] Furthermore, the emergence of drug resistance toward cisplatin in patients is a significant issue in the cancer therapy of patients. Niosomal cisplatin manufactured via reverse-phase evaporation exhibited a 1.5 -fold boost in cytotoxic activity against BT-20 breast cancer cells when compared against the unencapsulated drug [58] Antineoplastic effects of the niosomal formulations mentioned above are summarized in table 1.

Table 1: Antineoplastic effects exhibited by various niosomal formulations

\begin{tabular}{lll}
\hline Drug & Action shown & References \\
\hline Doxorubicin & Reduced proliferation of sarcoma cells & \\
Daunorubicin Hydrochloride & Shattered Dalton's ascetic lymphoma cells & Collected in high levels at the tumor site \\
Bleomycin & Improved antitumor activity against sarcoma & [40] \\
Methotrexate & Improved drug penetration in skin cancer treatment & {$[41]$} \\
5-Flourouracil & Improved antitumor activity against sarcoma & {$[42]$} \\
Vincristine & Improved cytotoxicity toward breast cancer cells \\
Tocotrienol & Improved cytotoxic and apoptotic effects toward ovarian cancer cells \\
Curcumin & Improved cytotoxicity toward cervical cancer cells \\
Curcumin and Doxorubicin Hydrochloride & Cytotoxicity toward melanoma cells & {$[44]$} \\
Arteminisin & High cytotoxicity against breast cancer cell line \\
Tamoxifen citrate & High cytotoxicity against human ovarian cancer and breast cancer cell lines \\
Mitoxantrone & Improved cytotoxicity toward breast cancer cells \\
Cisplatin & & {$[56]$} \\
\hline
\end{tabular}

\section{Ophthalmic drug delivery}

Bioadhesive-coated niosomal formulation of acetazolamide fabricated from Span 60, cholesterol stearylamine, or dicetyl phosphate shows more affinity toward decreasing the intraocular pressure when compared against marketed formulation (Dorzolamide); in contrast, the chitosan smeared niosomal formulation timolol maleate $(0.25 \%)$ demonstrates more activity in decreasing the intraocular pressure than marketed formulation with the reduced possibility of cardiovascular side effects [59].

\section{Delivery of peptide drugs}

Yoshida et al. [60] probed into the oral delivery of 9-desglycinamide, 8-arginine vasopressin as a model drug using niosome and by employing an in vitro intestinal loop model and enhanced the stability of peptide.

\section{Hemoglobin carriers}

Niosomes can be employed as hemoglobin carriers. The formulated niosomal suspension demonstrated a visible spectrum that can be superimposed over free hemoglobin. Vesicles are open for oxygen to travel inside the molecule and the hemoglobin dissociation curve can be altered similar to that of non-entrapped hemoglobin [61, 62].

\section{Antiviral drug delivery}

Furthermore, niosomes can deliver various antiviral drugs. Ruckmani and Sankar [63] prepared zidovudine encapsulated niosomes and assessed their entrapment efficiency and sustainability of drug release. The niosomes comprised Tween 80, Span 60, and cholesterol in various proportions. Niosomes comprising Tween 80 exhibited greater entrapment of zidovudine while the addition of dicetyl phosphate improved drug release for a longer duration. The drug outflow from Tween 80 containing formulations maintained at room temperature was substantial when compared to niosomes stored at $4{ }^{\circ} \mathrm{C}$ for $90 \mathrm{~d}$. Regardless, a pharmacokinetic study conducted on rabbits also verified that Tween 80 formulations prepared with dicetyl phosphate can be removed from systemic blood circulation in less than five h [64].

\section{Transdermal drug delivery}

Transdermal drug delivery is the delivery of drugs via the skin. The advantage of this delivery route is that transdermally administered drugs fail to undergo the first-pass metabolism; however, the penetration of drugs occurs slowly through the skin and this limitation can be resolved using niosome preparations. The mechanism obeyed by the niosomes for transdermal drug delivery is as follows.

- Diffusion via the stratum corneum layer.

- Water concentration in the skin is critical to this mechanism.

- The lipophilic drugs transverse the stratum corneum through various mechanisms such as aggregation, fusion, and adhesion.

- Nonionic surfactants improve the permeation thereby improving drug permeation via the skin $[65,66]$.

\section{Niosomes as drug carriers}

Niosomes have also been employed as carriers for iobitridol, a diagnostic agent used in X-ray imaging. Topical niosomes can perform several duties such as solubilization of the lipid matrix and act as local storage to provide sustained release of dermally active compounds, penetration boosters, or modifiers of the rate-limiting 
membrane barrier for the alteration of systemic drug absorption [67].

\section{Niosomes in vaccine delivery}

\section{A] Protein subunit vaccines}

The development of novel, safe, and efficacious vaccines, is a crucial aim for scientists worldwide. Subunit proteins or the deoxyribonucleic acid (DNA) of multiple organisms are less harmful than live organismbased vaccines, which may be relatively less clinically efficient. Adjuvants are being used in vaccines to improve the immunogenicity of the subunit vaccines via protection (i.e., preventing the destruction of the antigen in vivo) and upgraded targeting of these antigens to the desired antigen-presenting cells [68]. Brewer and Alexander [69] announced the first implementation of niosome antigen distribution for the immunization of Balb/c mice to develop resistance toward bovine serum albumin (BSA). They concluded that niosomes were possibly superior stimulators of the Th1 lymphocyte subset when compared against Freund's complete adjuvant, thus demonstrating very powerful stimulators of cellular immunity. Hassan et al. [70] announced enhanced immunogenicity with the herpes simplex virus 1 antigen encapsulated within l-mono palmitoyl glycerol (MP)/CHOL/DCP niosomes in mice. In contrast, only partial protection was observed against homologous (type 2 herpes simplex virus HSV-2) infection induced in the mice by the HSV2 antigen encapsulated niosomes [71], demonstrating the significance of composition in addition to the preparation methods for niosomal adjuvant formulations. Yoshioka et al. [72] prepared Span/CHOL/DCP niosomes comprising tetanus toxoid (TT) emulsified in an external oil phase to prepare a vesicle-in-water-in-oil (v/w/o) formulation. Initial studies on the system adopting cottonseed oil as the external oil phase in vivo, demonstrated improved immunological activity when compared to free antigen or vesicles. Murdan [73] entrapped BSA or hemagglutinin (HA) in the v/w/o emulsion and demonstrated via immunogenicity studies that in addition to the water-in-oil (w/o) gel that served as a control, the v/w/o gel exhibited immunoadjuvant abilities that intensified the primary and secondary antibody titers (of total IgG, IgG1, IgG2a, and IgG2b) to the HA antigen. Chambers et al. [74] announced an exclusive subcutaneous dose of killed Mycobacterium Bovis BCG in Brij ${ }^{\circledR}$ 52-containing nanoniosomes (NovasomeTM), which shielded guinea pigs from deadly tuberculosis. Vangala et al. [75] amalgamated three dissimilar protein antigens entrapped within positively charged niosomes synthesized from MP/CHOL/ $\alpha, \alpha^{\prime}-$ trehalose 6, 6'-dibehenate (TDB) or MP/CHOL/TDB/ dimethyldioctadecylammonium (DDA). Antigen entrapment within the niosomes enhanced the size of vesicles from sub microns to micrometers $(1-2.7 \mu \mathrm{m})$, which can be attributed to the high molecular weight of antigens and their stronger hydrophobic nature that causes protein aggregation in the hydrophobic zones of the vesicle bilayers while feasibly establishing a level of vesicle fusion or manipulating the packing positioning of the surfactants. Their conclusions recommend that both DDA-and MP-based vesicular systems can boost the immunogenicity of the subunit vaccines, primarily with the subunit antigen Ag85B-ESAT-6 immunized against tuberculosis, wherein the requirement of a high cell-mediated Th1 immune response is critical. Vangala et al. [76] also announced DDA formulations containing TDB, which demonstrated markedly improved hepatitis B surface antigen-specific splenocyte multiplication and induced cytokine synthesis in conjunction with a strong T-cell-based response, thus outlining formulations that can be useful for the supplementary assessment of their clinical value. Ferro and Stimson [77] adopted gonadotrophin-releasing hormone (GnRH) analog, GnRH-glycs, and joined two dissimilar carrier molecules and entrapped them within niosomes vesicular (NSV)-based formulations to attain immune-neutralization of GnRH in male Sprague-Dawley rats. They concluded that NSVs can be adopted as a safe immune adjuvant. Further, an altered GnRH peptide (CHWSYGLRPG-NH2) was linked to tetanus toxoid (TT), which was designed with unlike adjuvants such as C18E02/CHOL-/DCP niosomes [78]. When used with nanoniosomes, the sterilization effect demonstrated in the synthesis of IgG2b antibodies is not as promising as that attained with sustained-release poly-(lactideco-glycolide)/triacetin (PLGA) formulation. An encouraging immunization outcome was announced by Lezama-Davila [79] in C57BL/10 mice immunized with L. m. mexicana leishmanolysin (gp63). For the formulation of non-parenteral niosomal vaccines, Rentel et al. synthesized sucrose ester niosomes for the entrapment of ovalbumin and orally administered the vesicular formulations to Balb/c mice. Considerable enhancement in antibody titers was observed after oral vaccination with reduced hydrophilic vesicular formulation [80]. Chattaraj and Das [81] encapsulated hemagglutinin antigens from three divergent influenza A strains in Span 40 or 60 niosomes for nasal mucosal delivery. BSA-loaded niosomes fabricated from Span 60/Span 85/CHOL/stearylamine were smeared with an altered polysaccharide 0-palmitoyl mannan (OPM) for making them selective toward Langerhans' cells, the critical antigen-presenting cells existing in substantial amounts below the stratum corneum. Analyzing serum IgG titer and its subclasses (IgG2a/IgG1 ratio) induced a significantly greater serum IgG titer upon topical application of mannosylated niosomes as compared to topically applied alum adsorbed BSA $(\mathrm{p}<0.05)$. The mannosylated niosomes were also administered orally for showing oral mucosal immunization against TT. OPM coating was performed to shield the antigen-entrapped vesicles from bile salt dissolution activity and enzymatic breakdown in the gastrointestinal tract in addition to boosting the attraction of the antigens toward the antigen-presenting cells of Payer's patches [82]. Furthermore, Gupta et al. reported that, after secondary immunization, topically administered TT co-transferosomes could induce an immune response (anti-TT-IgG) equal to that obtained after performing intramuscular alum-adsorbed TT-based immunization. The immunity response of Span 85/CHOL niosomes was found to be shaky than that of the transferases [83].

\section{B] DNA vaccines}

DNA is a nucleic acid (biomolecule) that contains the genetic code specifying the biological development of all cellular forms of life and is often referred to as the molecule of heredity as it is responsible for the genetic propagation of all traits [84]. The fundamental principle of DNA vaccination is to induce immunity by transfecting host cells with plasmid DNA encoding the required antigen, contrary to the typical approach of injecting an antigen in the form of a protein or peptide [85]. Dengue ribonucleic aid has been discovered to possess more than five stereotypes [86]. DNA encapsulation in niosomes can be attributed to the shielding of genetic material in the biological environment, enhancing significant humoral as well as cell-mediated immune responses against the encoded antigen in immunized mice [87]. Perrie et al. [88] announced the encapsulation of nucleoprotein demonstrating the plasmid of the H3N2 influenza virus in NSVs followed by subcutaneous injection of the formulations that improved the immunization of treated mice relative to naked DNA. Vyas et al. prepared Span 85/CHOL niosomes containing DNA encoding HBsAg, which enhanced the serum anti HBsAg titer and the cytokine levels (IL-2 and IFN- $\gamma$ ) upon topical application in Balb/c mice, demonstrating the practical usefulness of the topical vesicular vaccine drug delivery system [89].

\section{CONCLUSION}

BCS is the base upon which drugs are classified into respective classes according to their solubility in water and permeability through the GIT; thus, through BCS, the problems of drugs can be identified potentially resolved. BCS employs various methods for determining solubility and permeability. Various drug delivery systems are available for BCS class II drugs, of which niosomes are more economical and safer carriers than liposomes. This review forms an insightful reference base for the various administration and preparation methods together with evaluation parameters and applications of niosomes in various fields of medicine.

\section{ACKNOWLEDGMENT}

The authors are grateful to the staff of H. K. College of Pharmacy for guiding towards the preparation of this manuscript.

\section{FUNDING}

Nil

\section{AUTHOR CONTRIBUTIONS}

All Authors have contributed equally. 


\section{CONFLICT OF INTERESTS}

None

\section{REFERENCES}

1. Amidon GL, Lennernas H, Shah VP, Crison JR. A theoretical basis for a biopharmaceutics drug classification: the correlation of in vitro drug product dissolution and in vivo bioavailability. Pharm Res 1995;12:413-20.

2. Chi-Yuan W, Benet LZ. Predicting drug disposition via application of BCS: Transport/Absorption/Elimination interplay and development of a biopharmaceutics drug disposition classification system. Pharm Res 2005;22:11-23.

3. Kerns EH, Di L. Multivariate pharmaceutical profiling for drug discovery. Curr Top Med Chem 2002;2:87-98.

4. Mujoriya RZ, Dhamande K, Bodla RB. Niosomal drug delivery system-a review. Int J Appl Pharm 2011;3:7-10.

5. Rogerson A, Cummings J, Willmott N, Florence AT. The distribution of doxorubicin in mice following administration in niosomes. J Pharm Pharmacol 1988;40:337-42.

6. Baillie AJ, Coombs GH, Dolan TF. Non-ionic surfactant vesicles, niosomes, as delivery system for the anti-leishmanial drug, sodium stibogluconate. J Pharm Pharmacol 1986;38:502-5.

7. Khandare JN, Madhavi G, Tamhankar BM. Niosomes novel drug delivery system. Eastern Pharm 1994;37:61-4.

8. Raja Naresh RA, Chandrashekhar G, Pillai GK, Udupa N. Antiinflammatory activity of niosome encapsulated diclofenac sodium with tween-85 in arthritic rats. Ind J Pharmacol 1994;26:46-8

9. Chauhan S, Luorence MJ. The preparation of polyoxyethylene containing non-ionic surfactant vesicles. J Pharm Pharmacol 1989;41:6p.

10. Blazek Walsh AI, Rhodes DG. SEM imaging predicts the quality of niosomes from maltodextrin-based proniosomes. Pharm Res 2001;18:656-61.

11. Amidon GL, Lennernas H, Shah VP, Crison JR. A theoretical basis for a biopharmaceutics drug classification: the correlation of in vitro drug product dissolution and in vivo bioavailability. Pharm Res 1995;12:413-20.

12. Guidance for industry, "Waiver of in vivo bioavailability and bioequivalence studies for immediate release solid oral dosage forms based on a biopharmaceutics classification system," CDER/FDA; 2000.

13. Biopharmaceutics classification system guidance office of pharmaceutical science, CDER/FDA; 2006.

14. Ku MS. Use of the biopharmaceutical classification system in early drug development. AAPS J 2008;10:208-12.

15. CA Lipiniski, F Lombardo, BW Dominy, PJ Feeney. Experimental and computational approaches to estimate solubility and permeability in drug discovery and development settings. Adv Drug Delivery Rev 1997;23:3-25.

16. C Bevan, RS Lloyd. A high-throughput screening method for the determination of aqueous drug solubility using laser nephelometry in microtiter plates. Anal Chem 2000;72:1781-7.

17. Avdeef. High throughput measurements of solubility profiles. In: B Testa, $\mathrm{H}$ van de Waterbeemd, G Folkers, $\mathrm{R}$ Guy. eds. Pharmacokinetic optimization in drug research: biological, physiological, and computational strategies, verlag helvetica chimica acta, zurich; 2001. p. 305-26.

18. $\mathrm{P}$ Artursson, $\mathrm{K}$ Palm, $\mathrm{K}$ Luthman. Caco-2 monolayers in experimental and theoretical predictions of drug transport. Adv Drug Delivery Rev 1996;22:67-84.

19. PV Balimane, Y Han, S Chong. Current industrial practices of assessing permeability and p-glycoprotein interaction. AAPS J 2006;8:E1-13.

20. JT Doluisio, NF Billups, LW Dittert, ET Sugita, JV Swintosky. Drug absorption. I. An in situ rat gut technique yielding realistic absorption rates. J Pharm Sci 1969;58:1196-200.

21. JD Irvine, L Takahashi, K Lockhart, J Cheong, JW Tolan, HE Selick, et al. MDCK (Madin-darby canine kidney) cells: a tool for membrane permeability screening. J Pharm Sci 1999;88:28-33.

22. Handjani Vila RM, Ribier A, Rondot B, Vanlerberghie G. Dispersions of lamellar phases of non-ionic lipids in cosmetic products. Int J Cosmet Sci 1979;1:303.
23. Saraswathi TS, Mothilal M, Jaganathan MK. Niosomes as an emerging formulation tool for drug delivery-a review. Int J Appl Pharm 2019;11:7-15.

24. Diljyot K. Niosomes: a new approach to targeted drug delivery. Int J Pharm Phytopharmacol Res 2012;2:53-9.

25. Kazi KM, Mandal AS, Biswas N, Guha A, Chatterjee S, Behera M, et al. Niosome: a future of targeted drug delivery systems. J Adv Pharm Technol Res 2010;1:374.

26. Yeo PL, Lim CL, Chye SM, Ling AP, Koh RY. Niosomes: a review of their structure, properties, methods of preparation, and medical applications. Asian Biomed 2018;11:301-14

27. Jayaraman SC, Ramachandran C, Weiner N. Topical delivery of erythromycin from various formulations: an in vivo hairless mouse study. J Pharm Sci 1996;85:1082-4.

28. Blazek Welsh AI, Rhodes DG. SEM imaging predicts the quality of niosomes from maltodextrin-based proniosomes. Pharm Res 2001;18:656-61.

29. Fernandes AV, Pydi CR, Verma R, Jose J, Kumar L. Design, preparation and in vitro characterizations of fluconazole loaded nanostructured lipid carriers. Braz J Pharm Sci 2020;56:1-14.

30. Seth AK, Misra A, Umrigar D. Topical liposomal gel of idoxuridine for the treatment of herpes simplex: pharmaceutical and clinical implications. Pharm Dev Technol 2005;9:277-89.

31. Kumar A, Agarwal SP, Ahuja A, Ali J, Choudhry R, Baboot S. Preparation, characterization, and in vitro antimicrobial assessment of nanocarrier based formulation of nadifloxacin for acne treatment. Pharmazie 2011;66:111-4.

32. Yoshioka T, Sternberg B, Florence AT. Preparation and properties of vesicles (niosomes) of sorbitan monoesters (Span 20, 40, 60 and 80) and a sorbitan triester (Span 85). Int J Pharm 1994;105:1-6.

33. Tavano L, Aiello R, Ioele G, Picci N, Muzzalupo R. Niosomes from glucuronic acid-based surfactant as new carriers for cancer therapy: preparation, characterization and biological properties. Colloids Surf B 2014;118:7-13

34. Priprem A, Janpim K, Nualkaew S, Mahakunakorn P. Topical niosome gel of Zingiber cassumunar Roxb extract for antiinflammatory activity enhanced skin permeation and stability of compound D. AAPS PharmSciTech 2016;17:631-9.

35. W Hua, T Liu. Preparation and properties of highly stable innocuous niosome in span $80 / \mathrm{PEG} 400 / \mathrm{H}_{2} \mathrm{O}$ system. Colloids Surf A 2007;302:377-82.

36. Manosroi A, Ruksiriwanich W, Abe M, Sakai H, Manosroi W, Manosroi J. Biological activities of the rice bran extract and physical characteristics of its entrapment in niosomes by supercritical carbon dioxide fluid. J Supercrit Fluids 2010;54:137-44.

37. Manosroi A, Chutoprapat R, Abe M, Manosroi J. Characteristics of niosomes prepared by supercritical carbon dioxide (scCO2) fluid. Int J Pharm 2008;352:248-55.

38. Azmin MN, Florence AT, Handjani Vila RM, Stuart JF Vanlerberghe G, Whittaker JS. The effect of non-ionic surfactant vesicle (niosome) entrapment on the absorption and distribution of methotrexate in mice. J Pharm Pharmacol 1985;37:237-42.

39. Hunter CA, Dolan TF, Coombs GH, Baillie AJ. Vesicular systems (niosomes and liposomes) for delivery of sodium stibogluconate in experimental murine visceral leishmaniasis. J Pharm Pharmacol 1988;40:161-5.

40. Gandhi A, Sen SO, Paul A. Current trends in niosome as vesicular drug delivery system. Asian J Pharm Life Sci 2012;2:2231:4423.

41. Cummings J, Stuart JF, Calman KC. Determination of Adriamycin, adriamycinol and their 7-deoxy aglycones in human serum by high-performance liquid chromatography. J Chromatogr B 1984;311:125-33.

42. Udupa N, Chandraprakash KS, Umadevi P, Pillai GK. Formulation and evaluation of methotrexate niosomes. Drug Dev Ind Pharm 1993;19:1331-42.

43. Paolino D, Cosco D, Muzzalupo R, Trapasso E, Picci N, Fresta M. Innovative bola-surfactant niosomes as topical delivery systems of 5-fluorouracil for the treatment of skin cancer. Int J Pharm 2008;353:233-42. 
44. Parthasarathi G, Udupa N, Umadevi PI, Pillai G. Niosome encapsulated of vincristine sulfate: improved anticancer activity with reduced toxicity in mice. J Drug Target 1994;2:173-82.

45. Nesaretnam K, Guthrie N, Chambers AF, Carroll KK. Effect of tocotrienols on the growth of a human breast cancer cell line in culture. Lipids 1995;30:1139-43.

46. Fu JY, Tan DM, Er HM, Chen YS, Nesaretnam K. Tumor-targeted niosome as novel carrier for intravenous administration of tocotrienol. Asian J Pharm Sci 2016;11:79-80.

47. Vallianou NG, Evangelopoulos A, Schizas N, Kazazis C. Potential anticancer properties and mechanisms of action of curcumin. Anticancer Res 2015;35:645-51.

48. Xu YQ, Chen WR, Tsosie JK, Xie X, Li P, Wan JB. Niosome encapsulation of curcumin: characterization and cytotoxic effect on ovarian cancer cells. J Nanomater 2016 https://doi.org/10.1155/2016/6365295

49. Sharma V, Anandhakumar S, Sasidharan M. Self-degrading niosomes for encapsulation of hydrophilic and hydrophobic drugs: an efficient carrier for cancer multi-drug delivery. Mater Sci Engi C 2015;56:393-400.

50. Antimalaria coordinating research group. Antimalaria studies on qinghaosu. Chin Med J 1979;92:811-6.

51. Crespo Ortiz MP, Wei MQ. Antitumor activity of artemisinin and its derivatives: from a well-known antimalarial agent to a potential anticancer drug. J Biomed Biotechnol 2012. DOI:10.1155/2012/247597

52. Li Q, Weina PJ, Milhous WK. Pharmacokinetic and pharmacodynamic profiles of rapid-acting artemisinins in the antimalarial therapy. Curr Drug Ther 2007;2:210-23.

53. Dwivedi A, Mazumder A, Du Plessis L, Du Preez JL, Haynes RK, Du Plessis J. In vitro anti-cancer effects of artemisone nano-vesicular formulations on melanoma cells. Nanomedicine 2015;11:2041-50.

54. Cole MP, Jones CT, Todd ID. A new anti-oestrogenic agent in late breast cancer: an early clinical appraisal of ICI46474. Br J Cancer 1971;25:270.

55. Shaker DS, Shaker MA, Hanafy MS. Cellular uptake, cytotoxicity and in vivo evaluation of Tamoxifen citrate loaded niosomes. Int J Pharm 2015;493:285-94.

56. Tila D, Yazdani Arazi SN, Ghanbarzadeh S, Aroma S, Pourmoazzen Z. pH-sensitive, polymer-modified, plasma stable niosomes: promising carriers for anti-cancer drugs. EXCLI J 2015;14:21.

57. Li M, Tang Z, Zhang Y, Lv S, Li Q, Chen X. Targeted delivery of cisplatin by LHRH-peptide conjugated dextran nanoparticles suppresses breast cancer growth and metastasis. Acta Biomater 2015;18:132-43.

58. Kanaani L. Effects of cisplatin-loaded niosomal nanoparticles on BT-20 human breast carcinoma cells. Asian Pac J Cancer Prev 2017;18:365.

59. Aggarwal D, Kaur IP. Improved pharmacodynamics of timolol maleate from a mucoadhesive niosomal ophthalmic drug delivery system. Int J Pharm 2005;290:155-9.

60. Gregoriadis G. Targeting of drugs: implications in medicine. Lancet 1981;318:241-7.

61. Dufes C, Gaillard F, Uchegbu IF, Schätzlein AG, Olivier JC, Muller JM. Glucose-targeted niosomes deliver vasoactive intestinal peptide (VIP) to the brain. Int J Pharm 2004;285:77-85.

62. Moser P, Marchand AM, Labrude P, Handjani VRM, Vignerson C. Niosomes d'hémoglobine preparation, proprietes physicochimiques et oxyphoriques, stabilite. Pharma Acta Helv 1989;64:192-202.

63. Ruckmani K, Sankar V. Formulation and optimization of zidovudine niosomes. AAPS PharmSciTech 2010;11:1119-27.

64. Ruckmani K, Sankar V, Sivakumar M. Tissue distribution, pharmacokinetics and stability studies of zidovudine delivered by niosomes and proniosomes. J Biomed Nanotechnol 2010;6:43-51.

65. Carafa M, Santucci E, Lucania G. Lidocaine-loaded non-ionic surfactant vesicles: characterization and in vitro permeation studies. Int J Pharm 2002;231:21-32.

66. Rastogi V, Pragya PU. A brief view on antihypertensive drugs delivery through transdermal patches. Int J Pharm Sci Res 2012;3:1955.

67. Makeshwar KB, Wasankar SR. Niosome: a novel drug delivery system. Asian J Pharm Res 2013;3:16-20.
68. Obrenovic MM, Perrie Y, Gregoriadis G. Entrapment of plasmid DNA into niosomes: characterization studies. J Pharm Pharmacol 1998;50:155.

69. Brewer J, Alexander J. The adjuvant activity of non-ionic surfactant vesicles (niosomes) on the BALB/c humoral response to bovine serum albumin. Immunology 1992;75:570-5.

70. Hassan Y, Brewer J, Alexander J, Jennings R. Immune responses in mice induced by HSV-1 glycoproteins presented with ISCOMs or NISV delivery systems. Vaccine 1996;14:1581-9.

71. Mohamedi S, Brewer J, Alexander J, Heath A, Jennings R. Antibody responses, cytokine levels and protection of mice immunized with HSV-2 antigens formulated into NISV or ISCOM delivery systems. Vaccine 2000;18:2083-94.

72. Yoshioka T, Skalko N, Gursel M, Gregoriadis G, Florence A. A nonionic surfactant vesicle in-water-in-oil (v/w/o) system: potential uses in drug and vaccine delivery. J Drug Target 1995;2:533-9.

73. Murdan S, Gregoriadis G, Florence A. Sorbitan monostearate/ polysorbate 20 organogels containing niosomes: a delivery vehicle for antigens. Eur J Pharm Sci 1999;8:177-86.

74. Chambers MA, Wright DC, Brisker J, Williams A, Hatch G, Gavier Widen D, et al. A single dose of killed mycobacterium bovis BCG in a novel class of adjuvant (Novasome ${ }^{\mathrm{TM}}$ ) protects guinea pigs from lethal tuberculosis. Vaccine 2004;22:1063-71.

75. Vangala A, Kirby D, Rosenkrands I, Agger EM, Andersen P, Perrie Y. A comparative study of cationic liposome and niosome-based adjuvant systems for protein subunit vaccines: characterization, environmental scanning electron microscopy and immunization studies in mice. J Pharm Pharmacol 2006:58:787-99.

76. Vangala A, Bramwell VW, McNeil S, Christensen D, Agger EM, Perrie Y. Comparison of vesicle based antigen delivery systems for delivery of hepatitis B surface antigen. J Controlled Release 2007;119:102-10.

77. Ferro V, Stimson W. Investigation into suitable carrier molecules for use in an antigonadotrophin releasing hormone vaccine. Vaccine 1998;16:1095-102.

78. Ferro VA, Costa R, Carter KC, Harvey MJ, Waterston MM, Mullen $\mathrm{AB}$, et al. Immune responses to a GnRH-based anti-fertility immunogen, induced by different adjuvants and subsequent effect on vaccine efficacy. Vaccine 2004;22:1024-31.

79. Lezama Dávila CM. Vaccination of C57BL/10 mice against cutaneous leishmaniasis. Use of purified gp63 encapsulated into niosomes surfactants vesicles: a novel approach. Mem Inst Oswaldo Cruz 1999;94:67-70.

80. Rentel C, Bouwstra J, Naisbett B, Junginger H. Niosomes as a novel peroral vaccine delivery system. Int J Pharm 1999;186:161-7.

81. Chattaraj S, Das S. Physicochemical characterization of influenza viral vaccine loaded surfactant vesicles. Drug Delivery 2003;10:73-7.

82. Jain S, Vyas S. Mannosylated niosomes as an adjuvant-carrier system for oral mucosal immunization. J Liposome Res 2006; 16:331-45

83. Gupta P, Mishra V, Rawat A, Dubey P, Mahor S, Jain S, et al. Noninvasive vaccine delivery in transfersomes, niosomes and liposomes: a comparative study. Int J Pharm 2005;293:73-82.

84. Adhikari AN, Mahar KS. DNA targeted anthraquinone derivatives: an important anticancer agents. Int J Pharm Pharm Sci 2016;8:17-25

85. Hudu SA, Shinkafi SH, Shuaibu U. An overview of recombinant vaccine technology, adjuvants and vaccine delivery methods. Int J Pharm Pharm Sci 2016;8:19-24.

86. Gade $\mathrm{K}$, Thanushree $\mathrm{N}$. The dengue vaccines: assessment of future prospects, treatment, and vaccine challenges. Asian J Pharm Clin Res 2020;13:4-9.

87. Mahor S, Gupta PN, Rawat A, Vyas SP. A needle-free approach for topical immunization: antigen delivery via vesicular carrier system. Curr Med Chem 2007;14:2898-910.

88. Perrie Y, Barralet J, McNeil S, Vangala A. Surfactant vesiclemediated delivery of DNA vaccines via the subcutaneous route. Int J Pharm 2004;284:31-41.

89. Vyas S, Singh R, Jain S, Mishra V, Mahor S, Singh P, et al. Nonionic surfactant-based vesicles (niosomes) for non-invasive topical genetic immunization against hepatitis B. Int J Pharm 2005;296:80-6. 